\title{
Factores que afectan la calidad de vida en pacientes con cirugía de contorno corporal: estudio de cohorte
}

\author{
Ekaterina Troncoso O. ${ }^{1,2}$, Stefan Danilla E. ${ }^{1}$, Pedro Cuevas T. ${ }^{1}$, \\ Cristian Erazo C. ${ }^{1}$, Susana Benítez S. ${ }^{1}$, Patricio Andrades C. ${ }^{1}$, \\ Sergio Sepúlveda P. ${ }^{1}$ y María Inés Godoy A. ${ }^{2}$
}

Factors that have an impact on quality of life in patients with body contouring surgery: a cohort study

\begin{abstract}
Aim: To identify the factors that have an impact on the quality of life of patients with body contouring surgery. Materials and Method: A prospective cohort of 113 patients was studied. Sociodemographic characteristics, antropometric measures and variables related to the surgery were analized. The Body-Qol ${ }^{\circledR}$ instrument was aplied. Descriptive statistic, generalized estimated equation models and lineal and logistic regresions were used for the statistical analysis. Results: Improvement in the quality of life was found globally $(\mathrm{p}<0,0001)$ and in every domain of the scale. The patients with massive weight loss had worse scores pre- and post-operatively than the esthetic patients. A positive association between age and improvement on quality of life was found. Body mass index (BMI) was negatively associated with improvement of the score. The patients that had a lipoabdominoplasty had a major improvement in comparison with other techniques. Also, the extended resection pattern and Fleur de Lis pattern were associated with lesser improvement in the global score. Conclusion: Body contouring surgery improves quality of life significantly. The principal factors that have an impact on quality of life were age, BMI, massive weight loss and resection pattern.
\end{abstract}

Key words: quality of life; body contouring; health impact assessment.

\section{Resumen}

Objetivo: Identificar los factores determinantes de la mejoría de la calidad de vida en pacientes sometidos a cirugía de contorno corporal. Materiales y Método: Se estudió una cohorte prospectiva de 113 pacientes sometidos a cirugía de contorno corporal. Se estudiaron características sociodemográficas, mediciones antropométricas, variables relativas a la cirugía y se aplicó el instrumento Body-Qol ${ }^{\circledR}$. Para el análisis estadístico se utilizó estadística descriptiva, modelos de ecuaciones de estimación generalizada y modelos de regresión lineal y logística. Resultados: Se encontró mejoría en la calidad de vida de forma global ( $\mathrm{p}<0,0001)$ y por dominios. Los pacientes con pérdida masiva de peso tuvieron peores puntajes pre y postoperatorio, sin embargo, un delta de mejoría comparable con los pacientes estéticos. Dentro de los factores determinantes, existió una relación positiva entre la edad y la mejoría en la calidad de vida de forma global. El índice de masa corporal (IMC) se encontró asociado negativamente con la mejoría del puntaje global. Dentro de los factores de la cirugía, los pacientes sometidos a lipoabdominoplastía tuvieron una mayor mejoría con respecto a otras técnicas. Además, el patrón de resección ampliado y en flor de Lis se asoció a menor mejoría en el puntaje global. Conclusión: La cirugía de contorno corporal mejora la calidad de vida de forma significativa. Los principales factores determinantes de esta mejoría fueron la edad, el IMC, el antecedente de pérdida masiva de peso y el patrón de resección.

Palabras clave: calidad de vida; contorno corporal; evaluación del impacto en la salud.
'Departamento de Cirugía,

Hospital Clínico de la Universidad de Chile. Santiago, Chile. 2Programa de Magíster en Epidemiología, Facultad de Medicina, Universidad de los Andes. Santiago, Chile.

Recibido 19 de marzo de 2019, aceptado para publicación el 27 de septiembre de 2019.

Correspondencia a: Dra. Ekaterina Troncoso Olchevskaia

ekaterina.troncoso@gmail. com 


\section{Introducción}

La cirugía de contorno corporal, como son la liposucción, la abdominoplastía y los body-lifts (Figura 1), buscan modificar el contorno del cuerpo para lograr una armonía estética, acorde con los cánones de belleza actuales. Sin embargo, no todos los pacientes que buscan modificar el contorno de su cuerpo lo hacen por razones estéticas. El exceso de adiposidad y piel redundante, además de ser inestético, puede producir problemas tanto físicos como psicológicos, como lo son intértrigo, excoriaciones, baja autoestima, aislamiento social, e incluso, bajo desempeño laboral ${ }^{1,2}$. Sumado a esto, el aumento de los pacientes con pérdida masiva de peso posterior a la cirugía bariátrica ha producido un incremento significativo en los pacientes que buscan cirugía de contorno corporal ${ }^{3-5}$.

El objetivo subyacente de la cirugía de contorno corporal es mejorar la calidad de vida de los pacientes. La estimación de la calidad de vida es compleja y demanda la utilización de instrumentos debidamente validados ${ }^{6}$. Actualmente, el mejor método para medir de forma objetiva la calidad de vida, es a través de cuestionarios denominados PROM ( $\mathrm{Pa}$ tient Reported Outcomes Measures). Los PROM son cualquier reporte del estado de salud proveniente directamente del paciente, sin mediar interpretación por otra persona ${ }^{7}$.

El instrumento Body-Qol $\AA$ es un PROM específicamente diseñado y validado para medir la calidad de vida en pacientes sometidos a cirugía de contorno corporal $^{8}$. Además, se ha demostrado que esta mejoría se mantiene en el tiempo?.

Sin embargo, los factores relacionados con este cambio en la calidad de vida no han sido estudiados. El objetivo de este estudio es explorar los factores determinantes de cambio en la calidad de vida en pacientes sometidos a cirugía de contorno corporal, evaluando las características demográficas, antecedentes clínicos y factores relacionados a la cirugía de los pacientes.

\section{Materiales y Método}

\section{Diseño}

Se diseñó un estudio de cohorte prospectiva, conformada por todos los pacientes operados de cualquier técnica de cirugía de contorno corporal por el equipo de Cirugía Plástica y Reparadora del Departamento de Cirugía del Hospital Clínico de la Universidad de Chile, en el período entre el 1 de octubre de 2015 y el 30 de septiembre de 2016, que hayan aceptado participar en el estudio y hayan firmado el consentimiento informado.

Los criterios de inclusión para cirugía fueron todos los pacientes categoría ASA I y II ${ }^{10}$ con exámenes preoperatorios normales. Se excluyeron todos los pacientes con IMC superior a $27,9 \mathrm{~kg} / \mathrm{m}^{2}$ en caso de cirugía de contorno corporal estética y mayor a $29,9 \mathrm{~kg} / \mathrm{mts}^{2}$ en caso de cirugía de contorno corporal posterior a la pérdida masiva de peso. Además, se

Figura 1. Evaluación de resultados mediante la fotografía pre y postoperatoria en una paciente sometida a liposucción y remodelación glútea.

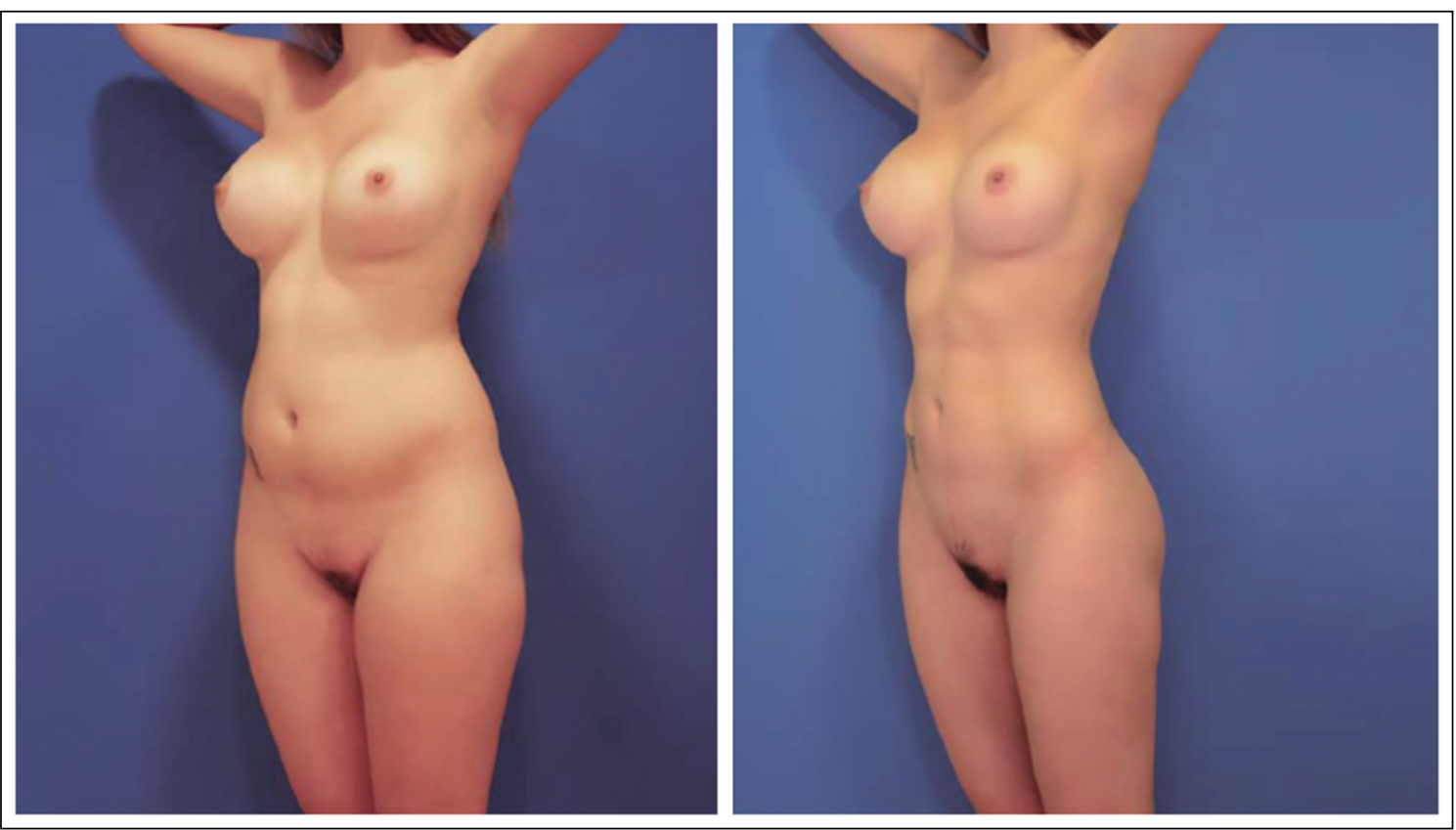


excluyeron todos los pacientes fumadores que no hayan suspendido el tabaco al menos un mes previo a la cirugía.

Este estudio fue aprobado por el Comité de Ética de la Universidad de Chile y fue realizado bajo los principios éticos propuestos en la declaración de Helsinki ${ }^{11}$.

\section{Variables}

La variable principal de resultado es el puntaje del instrumento Body-Qol ${ }^{\circledR}$ en el pre y postoperatorio. Este instrumento consta de 20 ítems distribuidos en 4 dominios (Tabla 1). Los ítems consisten en afirmaciones tipo Likert ${ }^{12}$ en las cuales el paciente evalúa su grado de acuerdo con la afirmación en una escala ordinal del 1 al 5. El puntaje obtenido puede fluctuar entre 20 (peor) y 100 (mejor). Para facilitar la interpretación del puntaje se ajustó la escala del 0 al 100 tanto en el puntaje global y por dominios.

Para evaluar la mejoría se consideró la diferencia entre el puntaje preoperatorio y el postoperatorio en forma total y por dominios.

Además, se estudiaron características sociodemográficas, antecedentes clínicos y variables relativas a la cirugía.

\section{Estadística}

Se utilizó estadística descriptiva para resumir y explicar las variables. La descripción de las variables continuas se realizó mediante promedio y desviación estándar para las variables con distribución simétrica, y mediana y rango intercuartílico (RIQ) con distribución asimétrica. La descripción de las variables nominales se realizó mediante frecuencia y porcentajes.

El análisis de la diferencia entre el puntaje pre y postoperatorio del Body-Qol ${ }^{\circledR}$ se realizó a través del test de Wilcoxon. Para determinar la asociación entre los factores estudiados y del puntaje pre y postoperatorio se utilizaron modelos de regresión lineal y logística, dependiendo de la naturaleza de la variable. Además, se utilizaron ecuaciones de estimación generalizada para evaluar la evolución del puntaje en el tiempo y relacionarlo con los factores determinantes.

Se rechazó la hipótesis nula con un nivel alpha de 1 en $20(p<0,05)$.

\section{Resultados}

\section{Descripción de la cohorte}

La cohorte fue conformada por 113 pacientes de un total de 137 que se sometieron a una cirugía de
Tabla 1. Instrumento Body-Qol ${ }^{\circledR}$. Consta de 20 ítems en forma de escala tipo Likert distribuidos en 4 dominios

\begin{tabular}{|ll|}
\hline Dominio & Ítem \\
Satisfacción & Me siento cómoda(o) mostrando mi guata \\
corporal & Me gusta mi guata cuando me la miro \\
& Mi guata se ve bella \\
& Mi guata está firme \\
& Me desagradan mis rollos \\
Vida sexual & Me siento cómodo(a) durante las relaciones sexuales \\
& Me siento seguro(a) sexualmente \\
& Me siento sexy estando desnudo(a) \\
& Evito que mi pareja me toque porque me siento feo(a) \\
& Evito que mi pareja me mire desnudo(a) \\
Autoimagen y & $\begin{array}{l}\text { Me siento bello(a) } \\
\text { autoestima }\end{array}$ \\
Me veo atractivo(a) estando vestido(a) \\
Las personas que me ven me encuentran atractivo(a) \\
Me siento seguro(a) de mi apariencia en un evento social \\
Me he sentido rechazado(a) por mis pares por mi apariencia \\
física
\end{tabular}

contorno corporal entre el 1 de octubre de 2014 y el 30 de septiembre de 2015 , es decir, el $82,48 \%$ de los pacientes participaron de este estudio. En el preoperatorio 113 pacientes completaron el instrumento Body-Qol $^{\circledR}$ (los que conformaron la cohorte) y 93 pacientes en el postoperatorio, con un seguimiento de $82,30 \%$. La cohorte se constituyó por 1 hombre y 112 mujeres, el $13,27 \%(n=15)$ de los pacientes tenían una cirugía bariátrica previa ( 5 bypass gástrico y 10 gastrectomía en manga) y una persona tenía una pérdida masiva de peso posterior a cambios en el estilo de vida; el resto de los pacientes $(n=97)$ se sometieron a la cirugía por razones estéticas. En promedio los pacientes tenían 38,52 $\pm 8,12$ años. El índice de masa corporal (IMC) fue de 25,21 $\pm 2,26$ $\mathrm{kg} / \mathrm{mts}^{2}$, teniendo menores IMC los pacientes operados por motivos estéticos $\left(25,08 \mathrm{~kg} / \mathrm{mts}^{2}\right)$ en contraste con los pacientes con cirugía bariátrica previa $\left(26,05 \mathrm{~kg} / \mathrm{mts}^{2} ; \mathrm{p}=0,03\right)$. En general eran pacientes sanos, sin embargo, el $26,54 \%$ de los pacientes eran fumadores, y el 11,50\% tenían el diagnóstico de depresión $\mathrm{y} / \mathrm{o}$ ansiedad. Los pacientes con antecedente de cirugía bariátrica tuvieron mayor proporción de 


\section{ARTÍCULO ORIGINAL}

diagnósticos de depresión $(\mathrm{p}=0,003)$ e hipertensión arterial (HTA) $(p=0,01)$ en contraste a los pacientes operados por motivos estéticos (Tabla 2).

El procedimiento más frecuentemente realizado fue lipoabdominoplastía en $96(84,96 \%)$ de los pacientes. Los pacientes con antecedente de pérdida masiva de peso presentaron una mayor proporción de procedimientos extensos, como lower body lift $(\mathrm{p}<0,0001)$ y abdominoplastía sin liposucción $(\mathrm{p}<0,0001)$. El patrón de resección más comúnmente usado fue en losanjo $(66,67 \%)$, seguido del patrón de Lockwood ${ }^{13}$ (18,28\%). Asimismo, los pacientes con antecedente de pérdida masiva de peso presentaron más frecuentemente resecciones circunferenciales $(p<0,0001)$ y en flor de lis $(\mathrm{p}=0,01)$ (Tabla 3$)$.

Tabla 2. Características de la cohorte general y diferencias entre pacientes estéticos y posterior a pérdida masiva de peso

\begin{tabular}{|lcccc|}
\hline Característica & $\begin{array}{c}\text { Cohorte completa } \\
\mathbf{n}=\mathbf{1 1 3}\end{array}$ & $\begin{array}{c}\text { Estéticos } \\
\mathbf{n = 9 7}\end{array}$ & $\begin{array}{c}\text { Post pérdida masiva de peso } \\
\mathbf{n = 1 6}\end{array}$ & $\begin{array}{c}\boldsymbol{p} \text { value (diferencia entre } \\
\text { estéticos y reconstructivos) }\end{array}$ \\
\hline Edad \pm DE (años) & $38,52 \pm 8,12$ & $38,83 \pm 7,50$ & $36,93 \pm 10,85$ & NS \\
Sexo (Mujeres:hombres) & $113: 1$ & $97: 0$ & $16: 1$ & NS \\
\hline Peso \pm DE (kg) & $63,10 \pm 12,33$ & $62,16 \pm 12,81$ & $68,5 \pm 7,04$ & 0,007 \\
Talla \pm DE (cm) & $156,01 \pm 27,15$ & $154,88 \pm 29,13$ & $162,5 \pm 7,10$ & NS \\
IMC \pm DE (kg/m²) & $25,23 \pm 2,26$ & $25,08 \pm 2,01$ & $26,05 \pm 3,27$ & 0,02 \\
Tabaquismo n (\%) & $30(26,54)$ & $26(26,80)$ & $4(25,00)$ & NS \\
\hline Depresión n (\%) & $10(8,85)$ & $6(5,31)$ & $4(25,00)$ & 0,003 \\
Ansiedad n (\%) & $9(7,96)$ & $7(7,22)$ & $2(12,5)$ & NS \\
\hline HTA n (\%) & $4(3,54)$ & $2(2,06)$ & $2(12,5)$ & 0,01 \\
\hline DM2 n (\%) & 0 & 0 & 0 & - \\
\hline Hipotiroidismo n (\%) & $6(5,31)$ & $6(6,19)$ & 0 & NS \\
\hline
\end{tabular}

$\mathrm{DE}=$ Desviación estándar. $\mathrm{NS}=$ No significativo.

Tabla 3. Tipo de cirugía realizada y patrón de resección en la cohorte completa y en pacientes estéticos y postpérdida masiva de peso

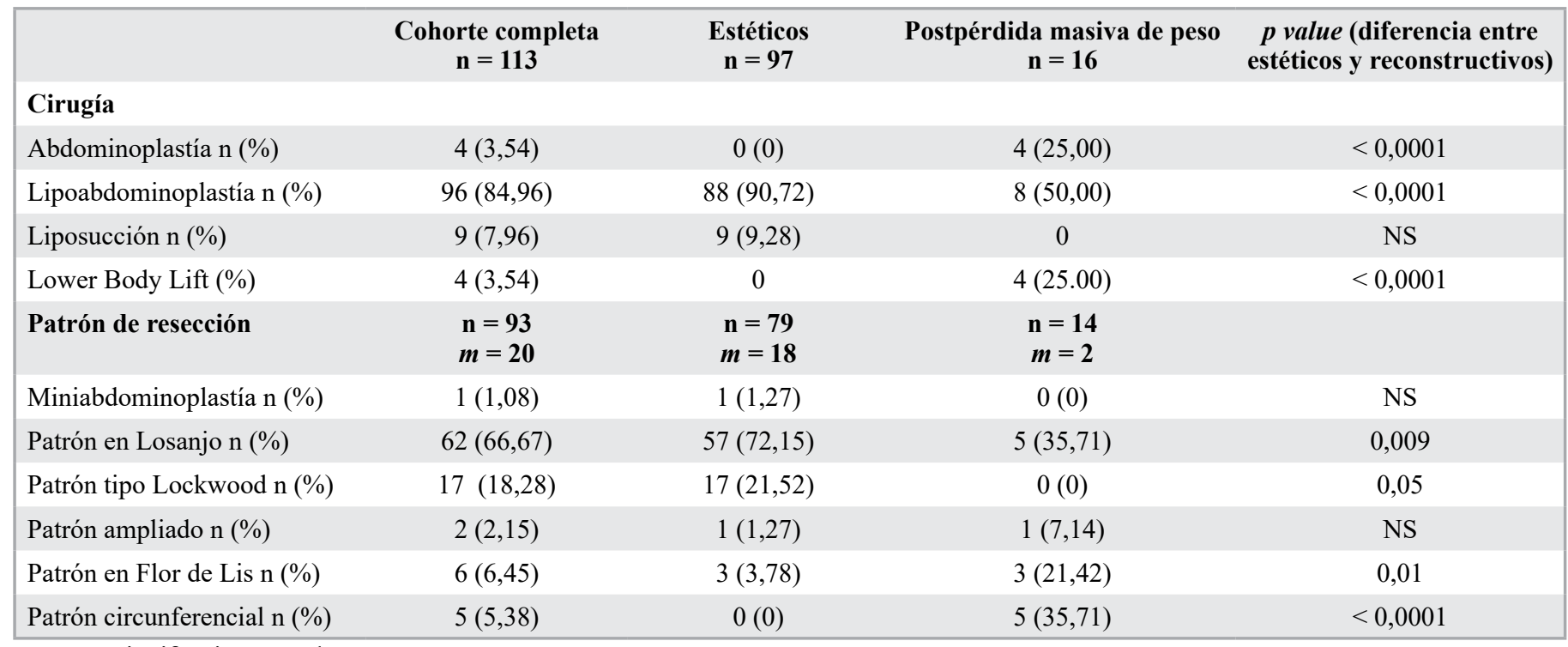

$\mathrm{NS}=$ No significativo. $m=$ datos $m$ missing . 


\section{Calidad de vida medida con el instrumento Body-Qol ${ }^{\circledR}$}

El instrumento Body-Qol ${ }^{\circ}$ se aplicó dentro de las $24 \mathrm{~h}$ previas a la cirugía y en el seguimiento postoperatorio entre el primer y décimo mes postoperatorio. La mediana de seguimiento fue de 3,1 meses (RIQ 3-6 meses). La puntuación general del Body-Qol ${ }^{\circledR}$ en el preoperatorio fue de 43,28 $\pm 14,53$ puntos y en el postoperatorio de $85,97 \pm$ 14,54 puntos. Esta diferencia fue estadísticamente significativa con un $\mathrm{p}<0,001$ (Figura 2). Además, hubo mejoría estadísticamente significativa en todos los dominios.

Al estratificar la cohorte en pacientes estéticos y con pérdida masiva de peso, encontramos menores puntajes en la cohorte con pérdida masiva de peso, tanto en el preoperatorio (37,94 versus 44,25 respectivamente; $\mathrm{p}=0,04)$ como en el postoperatorio (75,90 versus 87,24 respectivamente; $\mathrm{p}=0,01)$, sin embargo, la diferencia de mejoría fue similar en ambos grupos $(39,93$ versus 43,$79 ; p=0,33)$ (Figura 3).

\section{Calidad de vida y características de los pacientes}

Se encontró una tendencia de mayores puntajes postoperatorios con la edad $(\mathrm{p}=0,02)$. Además, se encontró asociación positiva entre la diferencia en la calidad de vida y la edad $(\mathrm{p}=0,025)$, la que se mantuvo en el modelo multivariado. Esta tendencia se observó en los dominios "Satisfacción Corporal" $(\mathrm{p}=0,02)$ y "Síntomas Físicos" $(\mathrm{p}=0,001)$ (Tabla 4). El dominio "Vida Sexual" tendió a la disminución a mayor edad $(\mathrm{p}=0,01)$ (Figura 4). El peso no se encontró asociado al cambio en la calidad de vida, sin embargo, el IMC se encontró asociado negativamente con la mejoría del puntaje global $(\mathrm{p}=0,02)$ (Figura 5). Asimismo, el IMC influyó de forma negativa en la mejoría de los dominios " $\mathrm{Au}$ toimagen y Autoestima" $(\mathrm{p}=0,01) \mathrm{y}$ "Síntomas físi$\cos "(p=0,001)$, relación que se mantuvo al ajustar el modelo por el antecedente de pérdida masiva de peso ( $p=0,01$ y $p=0,006$, respectivamente).

Al analizar el cambio de puntaje según los antecedentes mórbidos del paciente se observa una menor mejoría en los pacientes con tabaquismo crónico, depresión, hipertensión e hipotiroidismo, sin embargo, estas diferencias no fueron estadísticamente significativas. Ningún paciente presentó diabetes mellitus. Al analizar cada dominio por separado, se encontró asociación entre una menor mejoría y el tabaquismo en el dominio "Satisfacción Corporal" $(\mathrm{p}=0,02)$. También se encontró una asociación negativa entre la mejoría en el dominio "Autoimagen y Autoestima" y la presencia de depresión $(\mathrm{p}=0,01)$.
Además, la presencia de trastorno ansioso se asoció a menor mejoría en los dominios "Vida Sexual" $(p=0,04)$ y "Autoimagen y Autoestima" $(p=0,02)$. La HTA también tuvo una asociación negativa en el dominio "Vida Sexual" $(p=0,03)$ (Tabla 4).

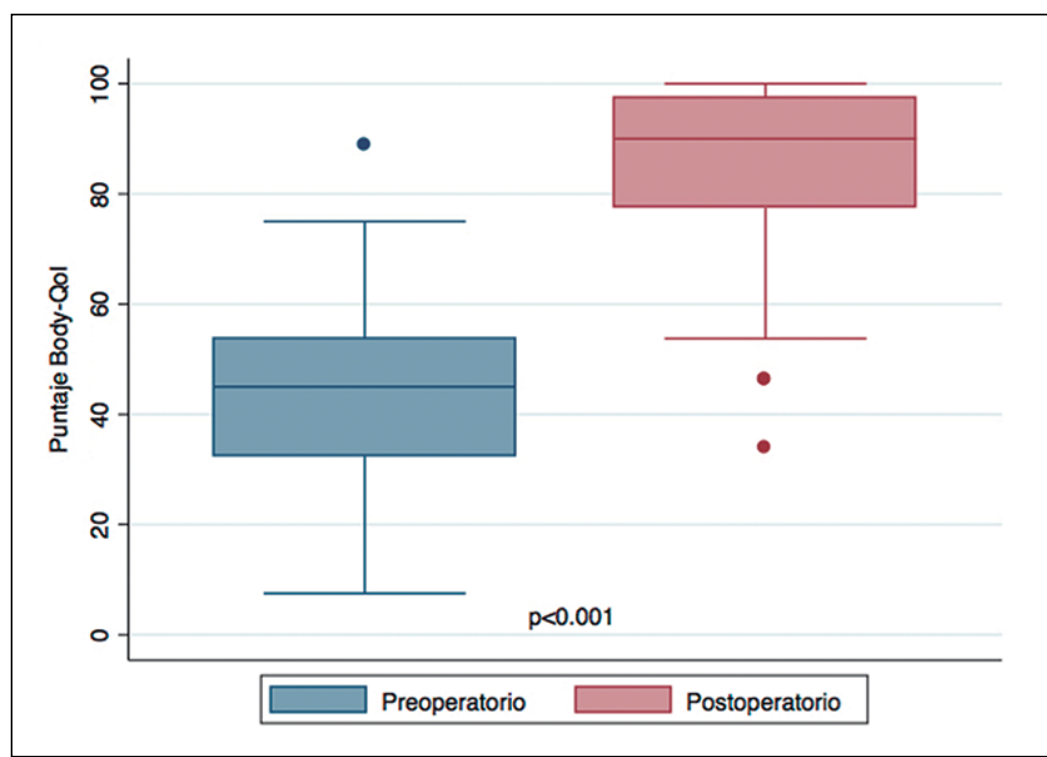

Figura 2. Puntaje Body-Qol ${ }^{\circledR}$ pre y postoperatorio. Se observa una diferencia estadísticamente significativa.

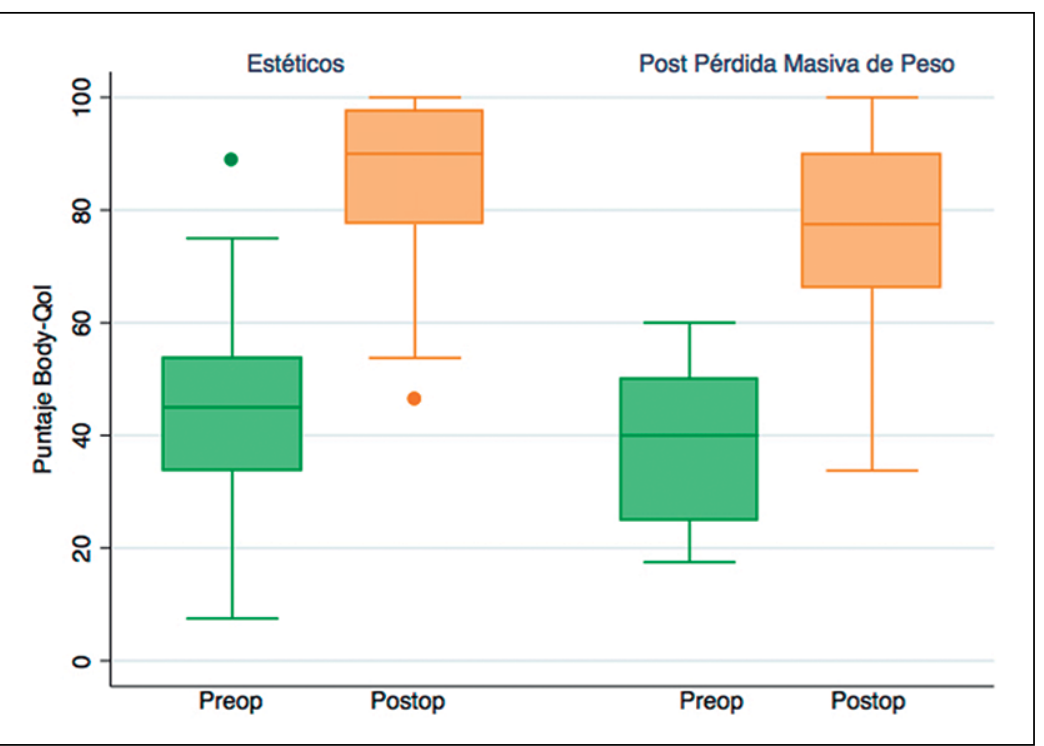

Figura 3. Cambio en la calidad de vida global en pacientes estéticos y con pérdida masiva de peso. Si bien los pacientes con pérdida masiva de peso tienen menores puntajes preoperatorios que los pacientes estéticos, tienen una mejoría en el puntaje similar, logrando puntajes postoperatorios elevados. 
Tabla 4. Asociación entre los antecedentes mórbidos y el cambio en el puntaje Body-Qol ${ }^{\circledR}$

\begin{tabular}{|c|c|c|c|c|c|c|c|c|c|c|c|c|c|c|c|}
\hline \multirow[b]{2}{*}{ Variable } & \multicolumn{3}{|c|}{ Escala global } & \multicolumn{3}{|c|}{ Satisfacción corporal } & \multicolumn{3}{|c|}{ Vida sexual } & \multicolumn{3}{|c|}{$\begin{array}{c}\text { Autoimagen y } \\
\text { autoestima }\end{array}$} & \multicolumn{3}{|c|}{ Síntomas físicos } \\
\hline & Coef. & $\begin{array}{l}\text { Stand. } \\
\text { error }\end{array}$ & $\begin{array}{c}p \\
\text { value }\end{array}$ & Coef. & $\begin{array}{l}\text { Stand. } \\
\text { error }\end{array}$ & $\underset{\text { value }}{p}$ & Coef. & $\begin{array}{l}\text { Stand. } \\
\text { error }\end{array}$ & $\underset{\text { value }}{p}$ & Coef. & $\begin{array}{l}\text { Stand. } \\
\text { error }\end{array}$ & $\underset{\text { value }}{p}$ & Coef. & $\begin{array}{l}\text { Stand. } \\
\text { error }\end{array}$ & $\begin{array}{c}p \\
\text { value }\end{array}$ \\
\hline Edad & 0,31 & 0,13 & 0,02 & 0,37 & 0,17 & 0,02 & $-0,18$ & 0,43 & 0,01 & 0,31 & 0,16 & NS & 0,63 & 0,38 & NS \\
\hline Peso & $-0,07$ & 0,08 & NS & $-0,04$ & 0,11 & NS & $-0,06$ & 0,13 & NS & $-0,06$ & 0,11 & NS & $-0,15$ & 0,11 & NS \\
\hline Talla & 0,05 & 0,06 & NS & 0,11 & 0,09 & NS & 0,001 & 0,1 & NS & 0,004 & 0,07 & NS & 0,11 & 0,11 & NS \\
\hline IMC & $-1,01$ & 0,53 & 0,05 & $-0,19$ & 0,69 & NS & $-0,36$ & 0,83 & NS & $-1,62$ & 0,64 & 0,01 & $-2,12$ & 0,65 & 0,001 \\
\hline Tabaquismo & $-4,84$ & 2,49 & 0,05 & $-6,93$ & 3,12 & 0,02 & $-5,16$ & 3,94 & NS & $-2,68$ & 3,12 & NS & $-3,57$ & 3,26 & NS \\
\hline Depresión & $-5,61$ & 3,73 & NS & $-5,67$ & 4,71 & NS & $-2,97$ & 5,84 & NS & $-11,02$ & 4,58 & 0,01 & $-2,33$ & 4,91 & NS \\
\hline Ansiedad & $-7,27$ & 3,91 & NS & $-1,96$ & 4,99 & NS & $-12,16$ & 6,03 & 0,04 & $-10,68$ & 4,84 & 0,02 & $-3,7$ & 5,16 & NS \\
\hline HTA & $-3,56$ & 6,18 & NS & $-9,71$ & 7,75 & NS & $-34,88$ & 16,03 & 0,03 & 6,29 & 7,53 & NS & $-4,44$ & 7,99 & NS \\
\hline Hipotiroidismo & 6,66 & 4,9 & NS & 7,19 & 6,13 & NS & 10,57 & 7,54 & NS & 2,84 & 6,09 & NS & 4,77 & 6,37 & NS \\
\hline
\end{tabular}

Coef $=$ Coeficiente. Stand. Error $=$ Error estándar. NS = no significativo.

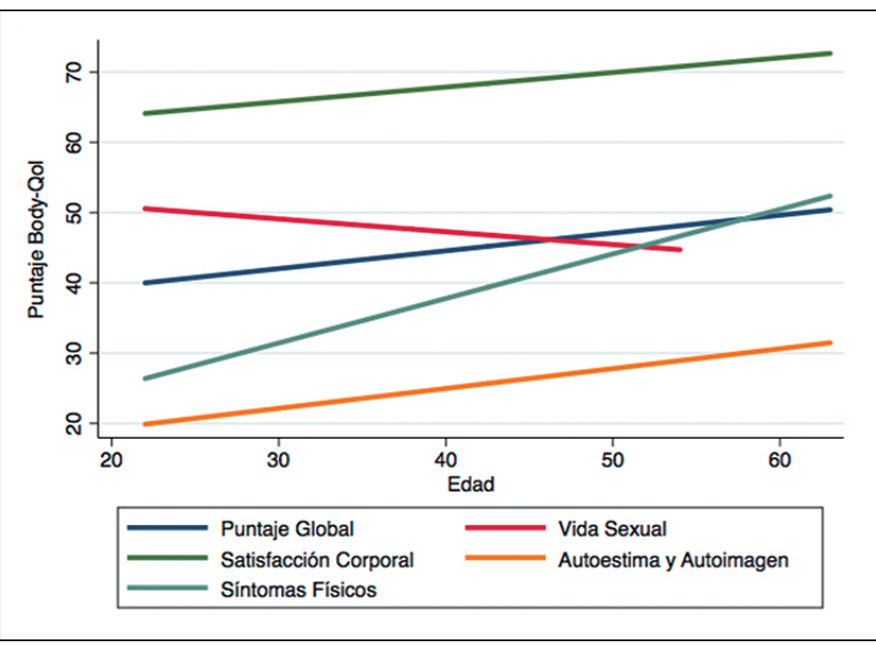

Figura 4. Valores ajustados de la diferencia de puntaje global y por dominios en relación a la edad. Se observa una clara tendencia positiva con la edad, excepto en el dominio Vida Sexual.

\section{Calidad de vida y características de la cirugía}

Según las variables de la cirugía, las pacientes con abdominoplastía y lipoabdominoplastía tuvieron una mayor mejoría en el puntaje con respecto a las otras técnicas $(\mathrm{p}=0,01)$ (Figura 7). En el análisis por dominios se encontró una mayor mejoría en las pacientes con lipoabdominoplastía en comparación con las otras técnicas en los dominios "Satisfacción corporal" $(p=0,0004)$ y "Síntomas físicos" $(p=0,0001)$ (Figura 8). El patrón de resección ampliado y en flor de lis se asociaron a menor mejoría en la calidad de vida global tanto en el modelo

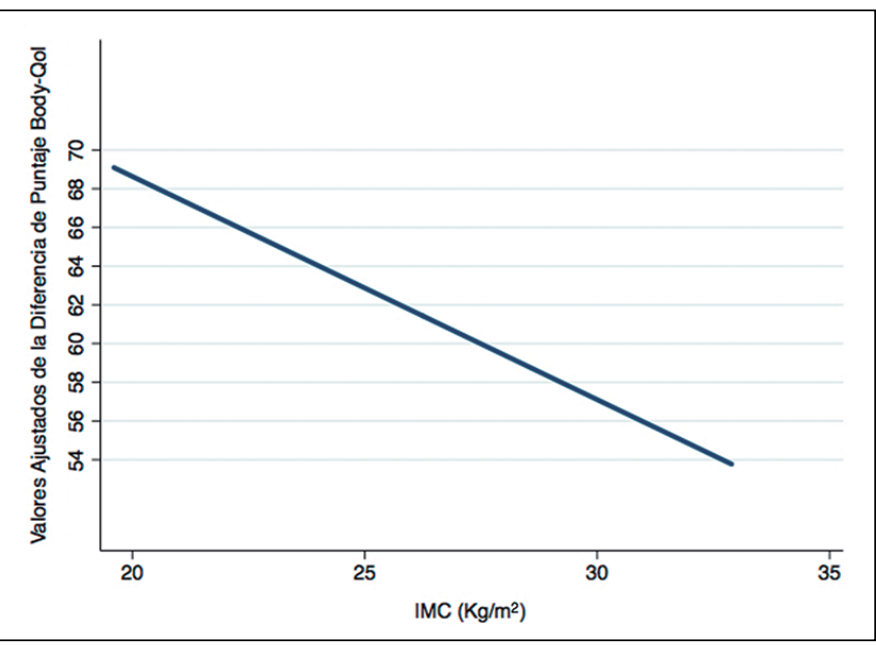

Figura 5. Valores ajustados de la diferencia de puntaje global en relación al IMC. Se observa una tendencia a menor mejoría en el puntaje a mayores IMC.

univariado como multivariado ( $p<0,0001$ y $p=0,02$ respectivamente). Por dominios, el patrón de resección ampliado se asoció a menor mejoría en la calidad de vida en todos los dominios, y el patrón en flor de lis a menor mejoría en el dominio "Autoimagen y Autoestima".

El volumen de la liposucción no tuvo asociación con la calidad de vida. En contraste, el peso del colgajo resecado tuvo una asociación negativa con la mejoría en la calidad de vida $(p=0,019)$, sin embargo, esta asociación se pierde en el modelo multivariado. 
ARTíCULO ORIGINAL

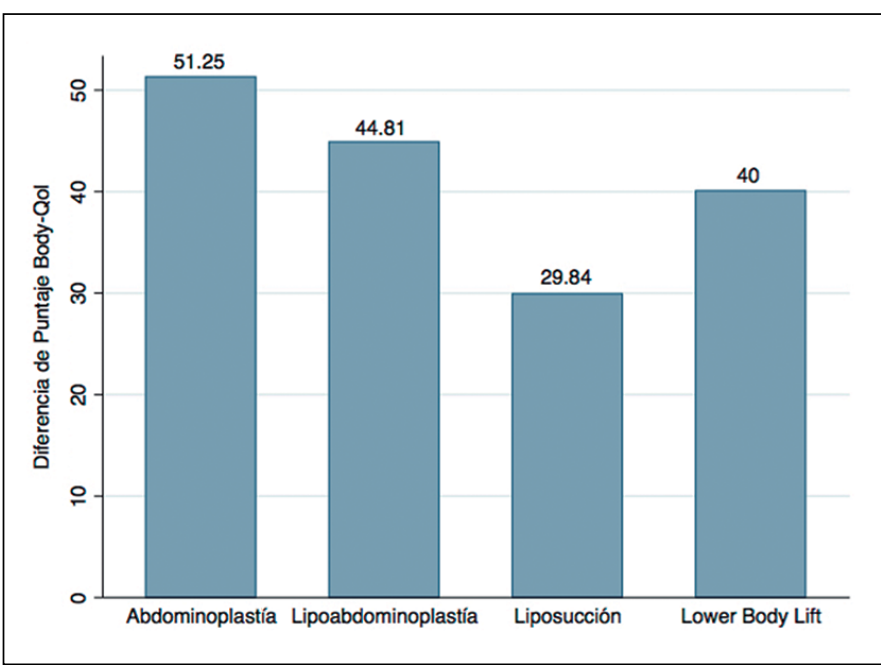

Figura 6. Diferencia entre el puntaje global preoperatorio y postoperatorio por tipo de cirugía.

\section{Discusión}

La evaluación de resultados a través de la estimación de la calidad de vida de los pacientes es un proceso fundamental, especialmente en cirugía plástica, en que la motivación para someterse a un procedimiento quirúrgico es diferente al padecimiento de una enfermedad. El Body-Qol ${ }^{\mathbb{}}$ es el primer instrumento diseñado y validado para evaluar la calidad de vida en cirugía de contorno corporal, tanto en pacientes estéticos como postbariátricos ${ }^{8}$. Estudios previos realizados por nuestro grupo sugieren una mejoría significativa en pacientes sometidos a cirugía de contorno corporal en el postoperatorio, sin embargo, los factores que determinan esta mejoría eran desconocidos.

La calidad de vida mejoró en forma significativa en forma global y en todos los dominios, tanto en la población estética como posterior a la pérdida masiva de peso. Esto es consistente con hallazgos publicados por otros grupos que han utilizado
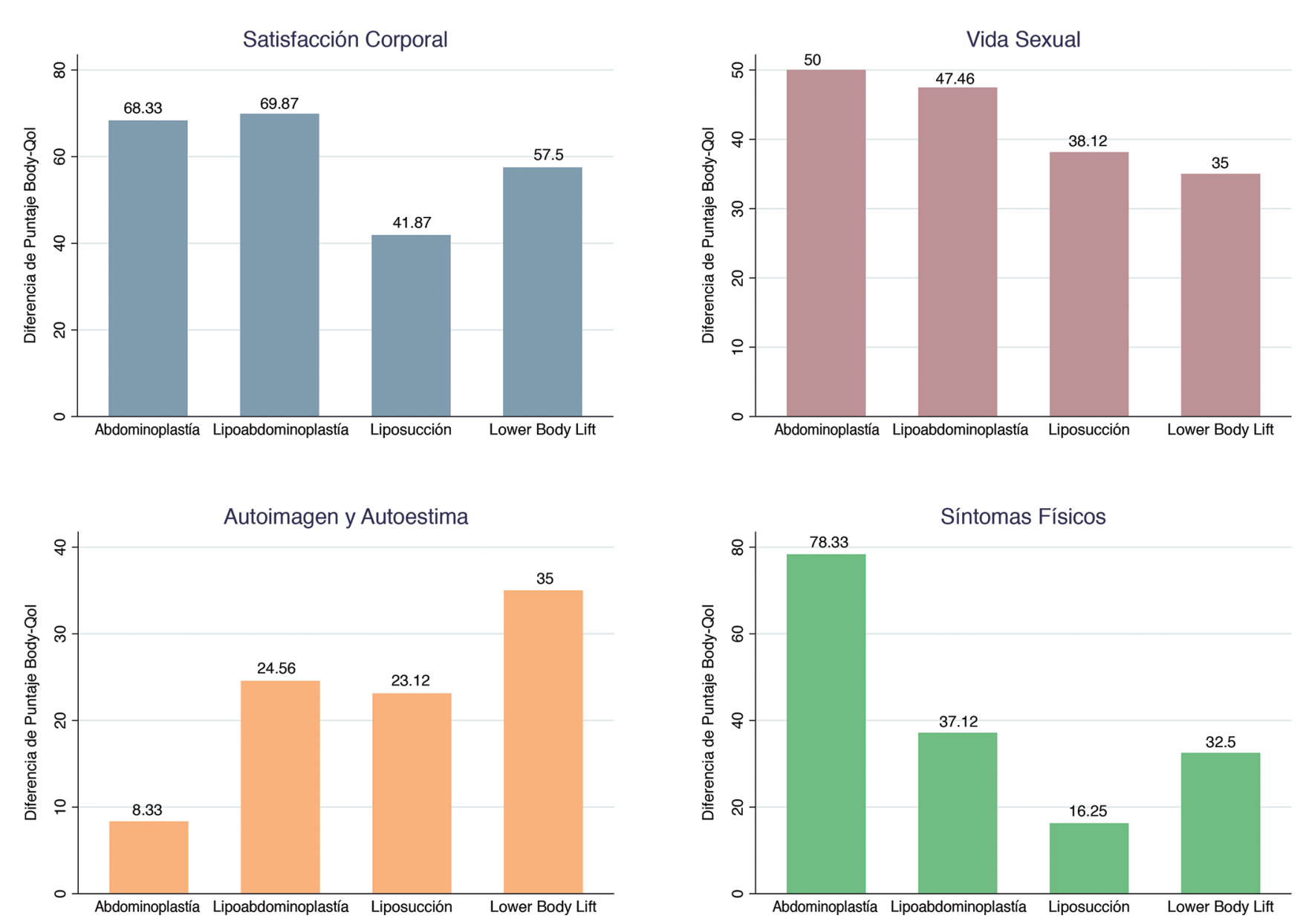

Figura 7. Diferencia entre el puntaje preoperatorio y postoperatorio por tipo de cirugía en cada dominio. 


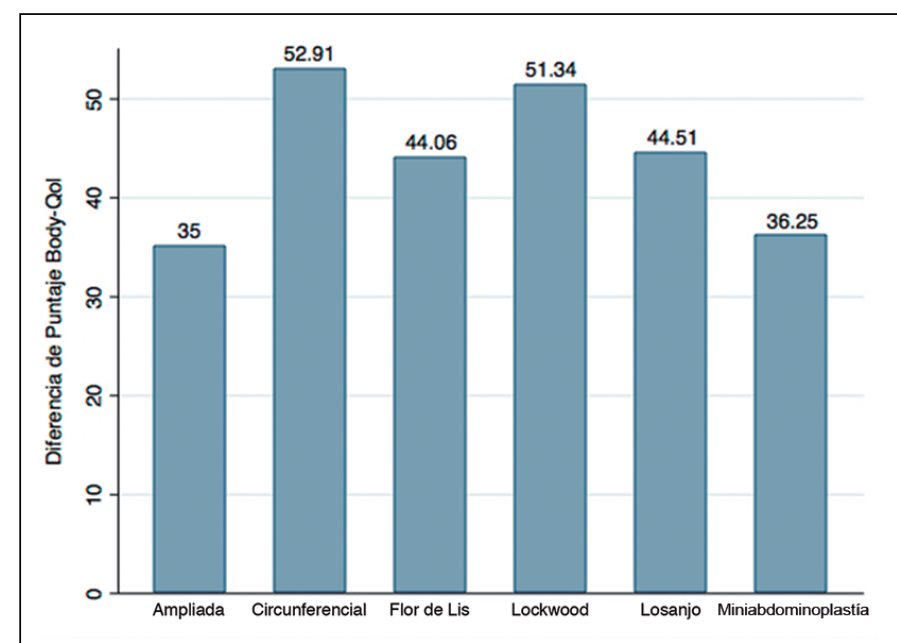

Figura 8. Diferencia entre el puntaje global preoperatorio y postoperatorio por patrón de resección. otros instrumentos para la medición de la calidad de vida ${ }^{14}, \mathrm{y}$ con resultados preliminares de nuestro grupo ${ }^{8}$.

Llama la atención que la población con el antecedente de pérdida masiva de peso presentó un incremento similar en la calidad de vida en comparación a la población estética, a pesar de presentar puntajes preoperatorios menores. Estudios realizados en población postbariátrica sometida a cirugía de contorno corporal demuestran asimismo un incremento sustantivo en la calidad de vida de estos pacientes ${ }^{15,16}$.

Dentro de los factores determinantes de la mejoría en la calidad destaca una mayor mejoría a mayor edad. Una publicación previa de nuestro grupo sugiere menor satisfacción con el contorno del cuerpo a mayores edades en las mujeres en población general $^{8}$, lo que habla que la población que se somete a cirugía de contorno corporal es diferente en este aspecto a la población general. Pensamos que la motivación para someterse a este tipo de cirugía está en relación al detrimento pronunciado en la calidad de vida derivada de la insatisfacción con el cuerpo, lo
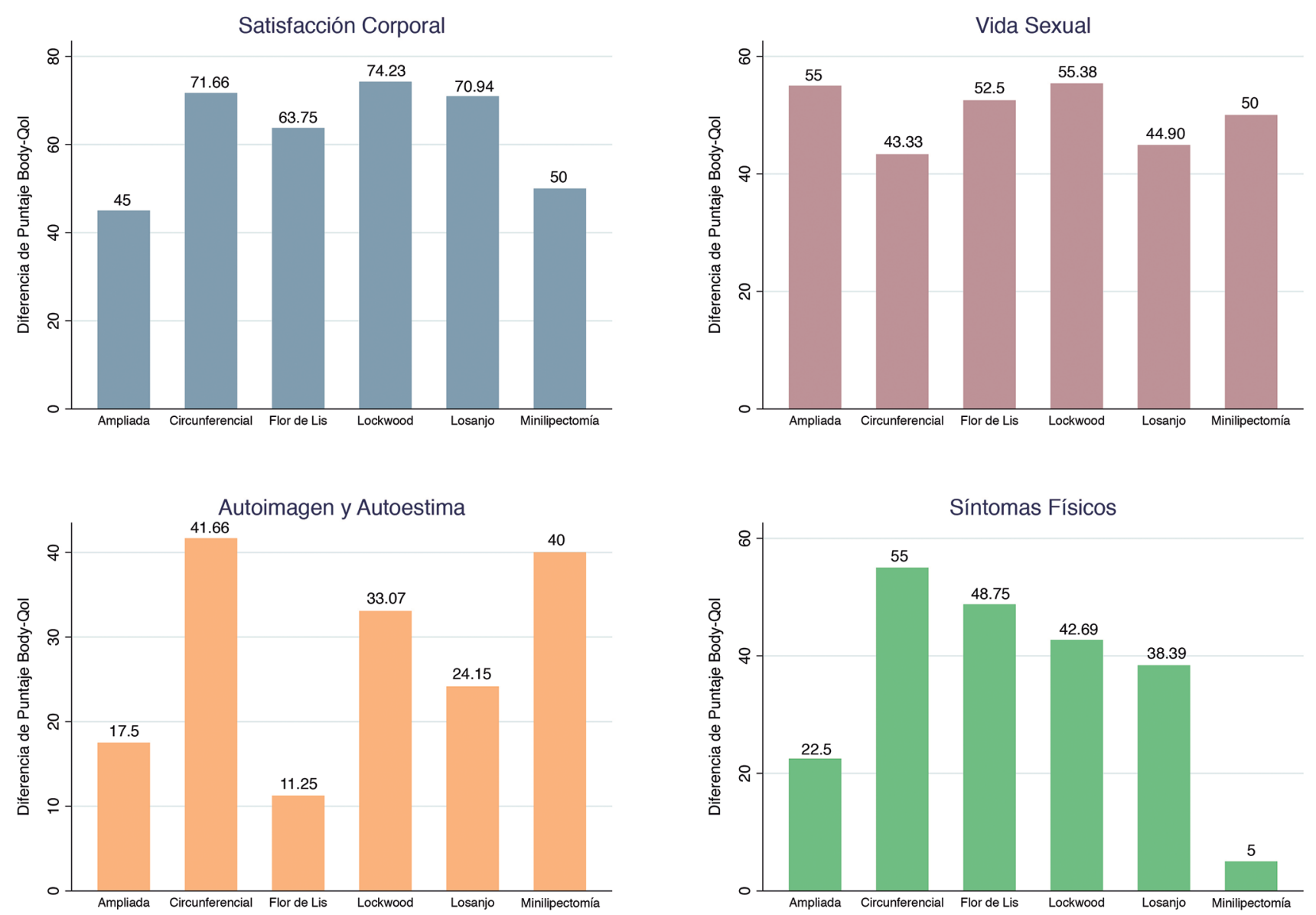

Figura 9. Diferencia entre el puntaje preoperatorio y postoperatorio por patrón de resección en cada dominio. 
que puede ocurrir a todas las edades. En contraste, las pacientes de mayores edades tuvieron menor mejoría en su vida sexual. Es posible que esto se encuentre relacionado a una causa compleja, no sólo derivada de la insatisfacción con el cuerpo. Flynn et al. (2016) en su artículo relacionado con la satisfacción sexual y su importancia sobre la calidad de vida realizado en 3.515 voluntarios, encontraron que la satisfacción con la vida sexual se encuentra relacionada al sexo, la edad, la raza y las comorbilidades en población general ${ }^{17}$.

Otro factor que afecta la calidad de vida fue el IMC, teniendo menor mejoría las pacientes con IMC mayores al momento de la cirugía, especialmente en los dominios relacionados a la autoimagen y autoestima y síntomas físicos. En una publicación Nigatu et al. (2016) encontraron una correlación entre menor calidad de vida y mayor grado de sobrepeso u obesidad, especialmente por empeoramiento de los síntomas físicos ${ }^{18}$. Este hallazgo, asociado a la menor tasa de complicaciones descritas con menores IMC, nos obliga a enfatizar en la normalización del peso de los pacientes previo a la cirugía, de modo de no solo realizar una cirugía más segura, sino que maximizar la calidad de vida postoperatoria.

El procedimiento más frecuentemente realizado fue la lipoabdominoplastía, el que se encontró asociado a una mayor mejoría en la calidad de vida, especialmente en los dominios relacionados con la satisfacción corporal y los síntomas físicos, mientras que las pacientes con liposucción tuvieron la menor mejoría en el puntaje. Esto se explica debido a que las pacientes con lipoabdominoplastía tuvieron mayores puntajes postoperatorios, y las pacientes con solo liposucción sola tenían mayores puntajes preoperatorios, acentuando esta diferencia. Además, patrones de resección más extensos se asociaron a menor mejoría en la calidad de vida, especialmente en el dominio relacionado a la autoimagen y autoestima, posiblemente por tratarse de pacientes con una mayor deformidad en el contorno y la presencia de cicatrices más extensas.

La mayor limitación de este estudio es la falta de representación de población masculina, por lo que estos resultados no son extrapolables a esta población en particular. Esto debido principalmente a que la mayor parte de nuestros pacientes que solicitan cirugía de contorno corporal son mujeres, proporción similar a la reportada en series internacionales ${ }^{19}$.

\section{Conclusiones}

La cirugía de contorno corporal mejora la calidad de vida de forma significativa, tanto en pacientes estéticos como posterior a pérdida masiva de peso. Esta mejoría se encontró en todos los dominios evaluados, incluyendo satisfacción corporal, vida sexual, autoimagen y autoestima, y síntomas físicos. Los principales factores determinantes de esta mejoría fueron la edad, el IMC, la presencia de depresión y ansiedad, el antecedente de pérdida masiva de peso y el patrón de resección. Las pacientes sometidas a cirugía de contorno corporal con mayor edad y menor IMC tuvieron una mayor mejoría en su calidad de vida. La presencia del diagnóstico de depresión o ansiedad tuvo un impacto negativo en la mejoría de la calidad de vida de nuestras pacientes. Los pacientes postbariátricos o con pérdida masiva de peso, si bien tuvieron menores puntajes postoperatorios, tuvieron un delta del puntaje en la calidad de vida similar a las pacientes estéticas. Asimismo, patrones de resección más extensos, como la abdominoplastía ampliada y en flor de lis tuvieron una menor mejoría.

\section{Responsabilidades éticas}

Protección de personas y animales. Los autores declaran que para esta investigación no se han realizado experimentos en seres humanos ni en animales.

Confidencialidad de los datos. Los autores declaran que en este artículo no aparecen datos de pacientes.

Conflictos de interés: no hay.

\section{Bibliografía}

1. de Brito MJA, Nahas FX, Barbosa MVJ, Dini GM, Kimura AK, Farah AB, et al. Abdominoplasty and its effect on body image, self-esteem, and mental health. Ann Plast Surg. 2010;65(1):5-10.

2. Klassen AF, Cano SJ, Scott A, Johnson
J, Pusic AL. Satisfaction and quality-oflife issues in body contouring surgery patients: a qualitative study. Obes Surg. 2012;22(10):1527-34.

3. Johnson EE, Simpson AN, Harvey JB, Lockett MA, Byrne KT, Simpson KN. Trends in bariatric surgery, 2002-2012: do changes parallel the obesity trend? Surg
Obes Relat Dis Off J Am Soc Bariatr Surg. 2016;12(2):398-404.

4. Altieri MS, Yang J, Park J, Novikov D, Kang L, Spaniolas K, et al. Utilization of Body Contouring Procedures Following Weight Loss Surgery: A Study of 37,806 Patients. Obes Surg. 2017;27(11):2981-7. 
5. Kitzinger HB, Abayev S, Pittermann A, Karle B, Kubiena H, Bohdjalian A, et al. The prevalence of body contouring surgery after gastric bypass surgery. Obes Surg. 2012;22:8-12.

6. Klassen AF, Cano SJ, Scott A, Tsangaris E, Pusic AL. Assessing Outcomes in Body Contouring. Clin Plastic Surg. 2014;41(4):645-54.

7. U.S. Department of Health and Human Services FDA Center for Drug Evaluation and Research, U.S. Department of Health and Human Services FDA Center for Biologics Evaluation and Research, U.S. Department of Health and Human Services FDA Center for Devices and Radiological Health. Guidance for industry: patient-reported outcome measures: use in medical product development to support labeling claims: draft guidance. Health Qual Life Outcomes. 2006;4:79.

8. Danilla S, Cuevas P, Aedo S, Domínguez C, Jara R, Calderón ME, et al. Introducing the Body-QoL ${ }^{\circledR}$ : A New Patient-Reported Outcome Instrument for Measuring Body Satisfaction-Related Quality of Life in Aesthetic and Post-bariatric Body Contouring Patients. Aesthetic Plast Surg. 2016;40(1):19-29.
9. Suijker J, Troncoso E, Pizarro F, Montecinos S, Villarroel G, Erazo C, et al. Long-Term Quality-of-Life Outcomes After Body Contouring Surgery: Phase IV Results for the Body-QoL ${ }^{\circledR}$ Cohort. Aesthet Surg J. 2018;38(3):279-88.

10. American Society of Anesthesiologists Physical Status Classification System [Internet]. 2019. Available from: https:// www.asahq.org/standards-and-guidelines/ asa-physical-status-classification-system

11. WMA Declaration of Helsinki - Ethical Principles for Medical Research Involving Human Subjects [Internet]. 2013 [cited 2016 Jun 8]. Available from: http://www. wma.net/es/30publications/10policies/b3/

12. Norman G. Likert scales, levels of measurement and the "laws" of statistics. Adv in Health Sci Educ. 2010;15(5):62532.

13. Lockwood T. High-lateral-tension abdominoplasty with superficial fascial system suspension. Plast Reconstr Surg. 1995;96(3):603-15.

14. Poulsen L, Klassen A, Rose M, Roessler KK, Juhl CB, Støving RK, et al. PatientReported Outcomes in Weight Loss and Body Contouring Surgery: A CrossSectional Analysis Using the BODY-Q. Plast Reconstr Surg. 2017;140(3):491-
500.

15. Monpellier VM, Antoniou EE, Aarts EO, Janssen IMC, Jansen ATM. Improvement of Health-Related Quality of Life After Roux-en-Y Gastric Bypass Related to Weight Loss. Obes Surg. 2017;27(5):1168-73.

16. Song P, Patel NB, Gunther S, Li C-S, Liu Y, Lee CYG, et al. Body Image \& Quality of Life: Changes With Gastric Bypass and Body Contouring. Ann Plast Surg. 2016;76:S216-21.

17. Flynn KE, Lin L, Bruner DW, Cyranowski JM, Hahn EA, Jeffery DD, et al. Sexual Satisfaction and the Importance of Sexual Health to Quality of Life Throughout the Life Course of U.S. Adults. J Sex Med. 2016;13(11):1642-50.

18. Nigatu YT, Reijneveld SA, de Jonge $\mathrm{P}$, van Rossum E, Bültmann U. The Combined Effects of Obesity, Abdominal Obesity and Major Depression/Anxiety on Health-Related Quality of Life: the LifeLines Cohort Study. PloS One. 2016;11(2):e0148871.

19. American Society of Plastic Surgery. 2017 Plastic Surgery Statistics. 2017; Available from: https://www.plasticsurgery.org/ documents/News/Statistics/2017/plasticsurgery-statistics-full-report-2017.pdf. 